\title{
Association between paraoxonase gene and stroke in the Han Chinese population
}

\author{
Guojun Zhang ${ }^{1 \dagger}$, Wenjin $\mathrm{Li}^{2 \dagger}$, Zhiqiang $\mathrm{Li}^{2}$, Hong Lv ${ }^{1}$, Yonghong Ren ${ }^{3,4}$, Ruimin $\mathrm{Ma}^{1}$, Xiaohong $\mathrm{Li}^{3,4}$, Xixiong Kang ${ }^{1}$, \\ Yongyong Shi ${ }^{2^{*}}$ and Yimin Sun ${ }^{3,4^{*}}$
}

\begin{abstract}
Background: The human paraoxonase (PON) gene family has three isoforms: PON1, PON2 and PON3. These genes are implicated as potential risk factors of cerebrovascular disease and can prevent oxidative modification of low-density lipoproteins and atherosclerosis. This study evaluated the association between the genetic variants of all three PON genes and the risks of total stroke, ischemic stroke and hemorrhagic stroke in the Han Chinese population.

Methods: A total of 1016 subjects were recruited, including 508 healthy controls and 498 patients (328 with ischemic stroke and 170 with hemorrhagic stroke). A total of 11 single nucleotide polymorphisms (SNPs) covering the PON genes were genotyped for statistical analysis. Two of the 11 SNPs (rs662 and rs854560) were contextualized in a meta-analysis of ischemic stroke.

Results: The presence of rs705381 (-162) in the promoter region of PON1 was significantly associated with total stroke $\left(P_{\text {adjusted }}=0.0007, O R=0.57[95 \% \mathrm{Cl}=0.41-0.79]\right)$ and ischemic stroke $\left(P_{\text {adjusted }}=0.0017, \mathrm{OR}=0.54[95 \% \mathrm{Cl}=\right.$ $0.37-0.79])$ when analyzed using a dominant model, but was not associated with hemorrhagic stroke. There was also a nominal association between rs854571 (-824) and total stroke. Meta-analysis demonstrated a significant nominal association between rs662 and ischemic stroke, but there was no evidence of an association between rs662 and ischemic stroke risk in a single site association study.
\end{abstract}

Conclusions: These findings indicate that polymorphisms of PON1 gene may be a risk factor of stroke.

Keywords: Polymorphisms, Paraoxanase gene, Hemorrhagic stroke, Ischemic stroke, Association

\section{Background}

Stroke is recognized as one of the leading causes of death and severe neurological disability worldwide. Ischemic and hemorrhagic stroke are the two primary subtypes [1]. Data from family-based studies [2], twin studies [3,4], and animal experiments [5,6] indicate that genetic factors play a major role in stroke. A small isolated group of strokes have previously been ascribed to single-gene disorders [7].

\footnotetext{
*Correspondence: shiyongyong@gmail.com; ymsun@capitalbio.com ${ }^{\dagger}$ Equal contributors

${ }^{2}$ Bio-X Institutes, Key Laboratory for the Genetics of Developmental and Neuropsychiatric Disorders (Ministry of Education), Shanghai Jiao Tong University, Shanghai 200030, China

${ }^{3}$ CapitalBio Corporation, 18 Life Science Parkway, Changping District, Beijing 102206, China

Full list of author information is available at the end of the article
}

Intermediate phenotypes of stroke are seen clinically. Atherosclerosis, as an intermediate phenotype of stroke, has been extensively investigated as a major underlying cause of cardio- and cerebrovascular disease [8-10]. There is also a strong inverse association between highdensity lipoprotein (HDL) levels and the development of atherosclerosis, and similar results have been found between low-density lipoprotein (LDL) peroxidation and the development of atherosclerosis [11,12].

The paraoxonase $(P O N)$ gene family comprises three isoforms, PON1, PON2 and PON3, located in 7q21.3-22.1 [13]. The 60 to $80 \%$ structural similarity among these three members accounts for their functional similarity $[13,14]$. All three isoforms have been implicated as candidate genes for atherosclerosis and cardiovascular diseases due to their ability to attenuate lipid peroxidation, and due to their antioxidant and antiatherogenic effects [15-17].

\section{Biomed Central}


Low levels of PON activity are thought to increase the risk of atherosclerosis [18], and thereby contribute to a predisposition towards stroke, coronary artery disease (CAD) and vascular disorders in diabetes [19-21]. Other studies have demonstrated a positive association between single nucleotide polymorphisms (SNPs) in PON genes and stroke susceptibility [22-25], although conflicting results have been seen in different ethnic groups [26-28]. However, there are limited number of prospective studies validating the association between $P O N$ genes and the risk of stroke in the Han Chinese population [26,28-30].

A negative association has previously been demonstrated between SNPs in the coding region of PON1 and $P O N 2$, and the development of stroke. In this study we wanted to evaluate the levels of ischemic and hemorrhagic risk conferred by SNPs in the whole PON family in a large Chinese population. With this aim in mind, we conducted a case-control study in the Han Chinese population to evaluate the possible association of $P O N$ family genes with total stroke and its subtypes.

\section{Methods \\ Subjects}

The study sample included 508 healthy controls and 498 patients, including 328 with ischemic stroke and 170 with hemorrhagic stroke who presented consecutively to the Department of Neurology, Beijing Tiantan Hospital, between December 2010 and March 2011. The subjects were unrelated to one another and were recruited from the Han Chinese population.

Hemorrhagic stroke included hypertensive cerebral hemorrhage and subarachnoid hemorrhage. Patients with hemorrhage due to trauma, tumor, vascular malformation and coagulopathy were excluded. Ischemic stroke was defined as a sudden onset of focal or global neurologic deficit with signs and symptoms persisting for more than $24 \mathrm{~h}$. Patients with a history or occurrence of transient ischemic attack, cerebral embolism, cerebral trauma, cerebrovascular malformations, coagulation disorders, autoimmune diseases, tumors, peripheral vascular disease, or chronic infection diseases were excluded from the study.

All diagnoses were confirmed by brain computed tomography and/or magnetic resonance imaging. The brain images were independently assessed by a technologist and a physician.

Control subjects were recruited from the health examination department of the Beijing Tiantan Hospital. These subjects had no clinical or radiological evidence of stroke and other neurological diseases. They were also free from autoimmune disease, liver disease, nephrosis, and hematological disorders

Sex, age, total plasma cholesterol (TC), triglycerides (TG), HDL, and LDL cholesterol were documented on entry into the study. Potential vascular risk factors were evaluated, including hypertension, diabetes mellitus, atrial fibrillation, and ischemic heart disease. Hypertension was defined according to WHO/ISH criteria [31] as systolic blood pressure $\geq 140 \mathrm{mmHg}$ and/or diastolic pressure $\geq 90 \mathrm{mmHg}$ with concomitant use of antihypertensive medications Diabetes mellitus was defined as fasting plasma glucose $\geq 7.0 \mathrm{mmol} / \mathrm{L}$ or current treatment with anti-diabetic drugs.

The experimental protocol was approved by the Ethics Committee of the Beijing Tiantan Hospital. Written informed consent was obtained from all participants prior to entering the study.

\section{Genotyping}

Eleven single nucleotide polymorphisms (SNPs) were genotyped. These included: rs662 (Gln192Arg), rs13306698 (Arg160Gly), rs854560 (Leu55Met) in coding region of PON1; rs705379 (-107/-108), rs705381 (-160/-162), rs854571 (-824/-832), rs854572 (-907/-909) in the promoter of PON1; rs12026 (Ala148Gly) and rs7493 (Ser311Cys) of PON2, together with rs2074353 (located in intron) and rs1053275 (Ala99Ala) for PON3.

The SNPs were genotyped using the Sequenom Mass ARRAY platform (Sequenom, San Diego, CA) according to the iPLEX Gold Application Guide available at (http:// www.sequenom.com/sites/genetic-analysis/applications/ snp-genotyping). The genotyping analysis was undertaken according to the manufacturer's protocol, using recommended reagents in the iPLEX Gold SNP genotyping kit. Briefly, specific assays were designed using the Mass ARRAY Assay Design software package (v3.1). The process involved a locus-specific PCR reaction based on a locusspecific primer extension reaction. Residual nucleotides were dephosphorylated with SAP enzymes before undertaking the iPLEX GOLD primer extension reactions.

Table 1 Comparison of clinical variables between total strokes and control subjects

\begin{tabular}{lll}
\hline Variables & $\begin{array}{l}\text { Stroke cases } \\
(\mathbf{n}=\mathbf{4 9 8})\end{array}$ & $\begin{array}{l}\text { Control cases } \\
(\mathbf{n = 4 9 8 )}\end{array}$ \\
\hline Ischemic stroke, $\mathrm{n}$ & 328 & \\
\hline Hemorrhagic stroke, $\mathrm{n}$ & 170 & $56.48 \pm 4.55$ \\
\hline Age, years & $60.45 \pm 14.27^{*}$ & $140(28)$ \\
\hline Male, $\mathrm{n}(\%)$ & $142(28)$ & $4.36 \pm 1.33$ \\
\hline $\mathrm{TC}, \mathrm{mmol} / \mathrm{L}$ & $4.41 \pm 1.31$ & $1.56 \pm 1.26$ \\
\hline $\mathrm{TG}, \mathrm{mmol} / \mathrm{L}$ & $1.54 \pm 0.95$ & $1.28 \pm 0.27$ \\
\hline $\mathrm{HDL}, \mathrm{mmol} / \mathrm{L}$ & $1.10 \pm 0.28^{*}$ & $2.52 \pm 0.56$ \\
\hline $\mathrm{LDL}, \mathrm{mmol} / \mathrm{L}$ & $2.54 \pm 0.89$ & $310(62)$ \\
\hline Hypertension, $\mathrm{n}(\%)$ & $413(83)^{*}$ & $122(24)$ \\
\hline Diabetes, $\mathrm{n}(\%)$ & $130(26)$ & . \\
\hline
\end{tabular}

Data are shown as mean \pm standard deviation (SD) or as $n$ (\%). Abbreviations: TC, total cholesterol; TG, triglycerides; HDL, high-density lipoprotein; LDL, lowdensity lipoprotein. *Significant differences between cases and controls. 


\begin{tabular}{|c|c|c|c|c|c|c|c|c|c|c|c|}
\hline \multirow{2}{*}{ a } & & & & \multicolumn{5}{|c|}{$r^{2}$} & \multirow[b]{2}{*}{ rs1053275 } & \multirow[b]{2}{*}{ rs7493 } & \multirow[b]{2}{*}{ rs $1202 \epsilon$} \\
\hline & & rs 662 & rs13306698 & rs854560 & rs705381 & rs854571 & rs854572 & rs2074353 & & & \\
\hline \multirow{10}{*}{$\bar{n}$} & rs662 & - & 0.062 & 0.07 & 0.112 & 0.013 & 0.252 & 0.191 & 0.175 & 0.079 & 0.077 \\
\hline & rs13306698 & 0.974 & - & 0.005 & 0.017 & 0.183 & 0.073 & 0.028 & 0.022 & 0.025 & 0.025 \\
\hline & rs 854560 & 1 & 1 & - & 0.002 & 0.037 & 0.014 & 0.074 & 0 & 0.076 & 0.074 \\
\hline & rs705381 & 0.666 & 1 & 0.51 & - & 0.286 & 0.106 & 0.002 & 0 & 0.108 & 0.107 \\
\hline & rs 854571 & 0.125 & 0.897 & 0.646 & 0.977 & - & 0.366 & 0 & 0.053 & 0.042 & 0.042 \\
\hline & rs854572 & 0.744 & 0.925 & 0.646 & 0.967 & 1 & - & 0.202 & 0.141 & 0.069 & 0.065 \\
\hline & rs2074353 & 0.593 & 0.884 & 0.75 & 0.21 & 0.04 & 0.898 & - & 0.743 & 0.005 & 0.006 \\
\hline & rs1053275 & 0.656 & 0.903 & 0.22 & 0.041 & 0.669 & 0.865 & 0.996 & - & 0.047 & 0.047 \\
\hline & rs7493 & 0.457 & 1 & 0.642 & 0.404 & 0.302 & 0.629 & 0.263 & 0.924 & - & 0.997 \\
\hline & rs12026 & 0.449 & 1 & 0.637 & 0.402 & 0.299 & 0.613 & 0.277 & 0.924 & 1 & - \\
\hline
\end{tabular}

b

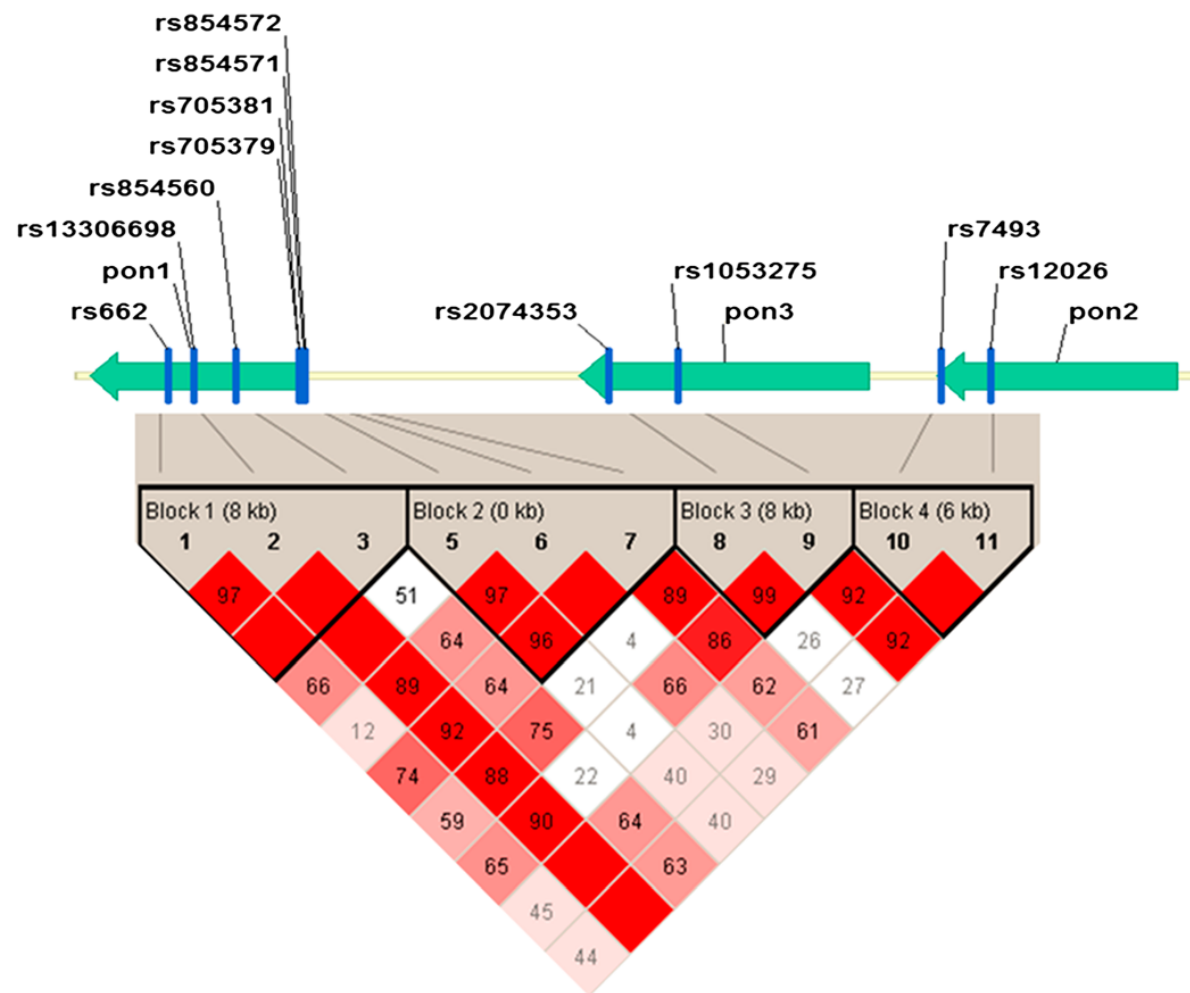

Figure 1 Linkage disequilibrium analysis of the ten SNPs investigated in healthy controls (a). Four blocks were identified using Haploview software: Block1 (rs854560-rs13306698-rs662); Block2 (rs854572-rs854571-rs705381); Block3 (rs1053275-rs2074353); Block4 (rs12026-rs7493) (b).

Following the single-base extension reactions the products were desalinated with Spectro CLEAN resin (Sequenom). A $10 \mathrm{~nL}$ aliquot of the desalinated product was spotted onto a 384-format Spectro CHIP with the Mass ARRAY Nanodispenser. Mass determination was carried out with the MALDI-TOF mass spectrometer and Mass ARRAY Type 4.0 software was used for data acquisition.

SNP genotypes were named using cluster analysis with a default parameter setting. Genotypes were further reviewed manually to correct classification errors caused by clustering artifacts.

\section{Statistical analysis}

Statistical analysis was undertaken using PLINK software (http://pngu.mgh.harvard.edu/ purcell/plink/) [32]. HardyWeinberg equilibrium tests (HWE) were performed for each SNP, and association tests were undertaken using additive, dominant, or recessive genetic models.

Logistic regression was used for risk stratification with or without covariate adjustments determined by significant differences between total stroke patients and controls (i.e. age, HDL, and hypertension). The model with the highest likelihood was considered to provide the best-fit genetic model for each SNP. Haplotype-based 
Table 2 Association between SNPs and total stroke using the additive, dominant, genotype, and the recessive models

\begin{tabular}{|c|c|c|c|c|c|c|c|c|}
\hline \multirow[t]{2}{*}{ SNP } & \multirow[t]{2}{*}{ Model } & \multirow{2}{*}{$\begin{array}{l}\text { Allele or } \\
\text { geno }\end{array}$} & \multirow[t]{2}{*}{ F_Stroke } & \multirow[t]{2}{*}{ F_Control } & \multicolumn{2}{|l|}{ T-Statistic } & \multicolumn{2}{|c|}{ Logistic Regression } \\
\hline & & & & & OR $(95 \% \mathrm{Cl})$ & $P_{\text {unadjusted }}$ & OR $(95 \% \mathrm{Cl})$ & $P_{\text {adjusted }}$ \\
\hline \multirow[t]{3}{*}{ rs 854571} & Additive & $C>T$ & 298/992 & $344 / 982$ & $0.79(0.65-0.96)$ & $1.71 \mathrm{E}-02$ & $0.80(0.65-0.98)$ & $3.30 \mathrm{E}-02$ \\
\hline & Dominant & $\mathrm{CC}+\mathrm{CT} / \mathrm{TT}$ & $253 / 496$ & $289 / 491$ & $0.73(0.57-0.94)$ & $1.33 \mathrm{E}-02$ & $0.75(0.57-0.99)$ & $3.96 \mathrm{E}-02$ \\
\hline & Recessive & $\mathrm{CC} / \mathrm{CT}+\pi$ & $45 / 496$ & $55 / 491$ & $0.79(0.52-1.20)$ & 2.69E-01 & $0.75(0.48-1.17)$ & 2.06E-01 \\
\hline \multirow[t]{3}{*}{ rs13306698 } & Additive & $A>G$ & $98 / 1016$ & $98 / 988$ & $0.97(0.71-1.31)$ & $8.31 \mathrm{E}-01$ & $1.00(0.71-1.40)$ & 9.99E-01 \\
\hline & Dominant & $\mathrm{AA}+\mathrm{AG} / \mathrm{GG}$ & $97 / 508$ & $96 / 494$ & $0.98(0.71-1.34)$ & $8.92 \mathrm{E}-01$ & $1.02(0.72-1.44)$ & $9.20 \mathrm{E}-01$ \\
\hline & Recessive & $\mathrm{AA} / \mathrm{AG}+\mathrm{GG}$ & $1 / 508$ & $2 / 494$ & $0.49(0.04-5.37)$ & 5.55E-01 & $0.39(0.03-5.06)$ & 4.74E-01 \\
\hline \multirow[t]{3}{*}{ rs854572 } & Additive & $C>G$ & $443 / 1004$ & $413 / 964$ & $1.05(0.88-1.26)$ & 5.69E-01 & $1.09(0.89-1.32)$ & $4.08 \mathrm{E}-01$ \\
\hline & Dominant & $\mathrm{CC}+\mathrm{CG} / \mathrm{GG}$ & $343 / 502$ & $324 / 482$ & $1.05(0.81-1.38)$ & 7.10E-01 & $1.11(0.82-1.48)$ & $5.04 \mathrm{E}-01$ \\
\hline & Recessive & $\mathrm{CC} / \mathrm{CG}+\mathrm{GG}$ & $100 / 502$ & $89 / 482$ & $1.10(0.80-1.51)$ & $5.62 \mathrm{E}-01$ & $1.13(0.80-1.61)$ & 4.93E-01 \\
\hline \multirow[t]{3}{*}{ rs7493 } & Additive & $C>G$ & $192 / 1016$ & $176 / 974$ & $1.06(0.84-1.33)$ & $6.32 \mathrm{E}-01$ & $1.00(0.78-1.29)$ & 9.89E-01 \\
\hline & Dominant & $\mathrm{CC}+\mathrm{CG} / \mathrm{GG}$ & $173 / 508$ & $163 / 487$ & $1.03(0.79-1.34)$ & $8.45 \mathrm{E}-01$ & $1.00(0.75-1.34)$ & $9.85 \mathrm{E}-01$ \\
\hline & Recessive & $\mathrm{CC} / \mathrm{CG}+\mathrm{GG}$ & $19 / 508$ & $13 / 487$ & $1.42(0.69-2.90)$ & $3.41 \mathrm{E}-01$ & $1.00(0.46-2.18)$ & 9.93E-01 \\
\hline \multirow[t]{3}{*}{ rs662 } & Additive & $G>A$ & $389 / 1014$ & $356 / 978$ & 1.08(0.91-1.29) & 3.86E-01 & 1.05(0.87-1.28) & 5.93E-01 \\
\hline & Dominant & $\mathrm{GG}+\mathrm{GA} / \mathrm{AA}$ & $303 / 507$ & $282 / 489$ & $1.09(0.85-1.40)$ & $5.02 \mathrm{E}-01$ & $1.05(0.80-1.39)$ & 7.31E-01 \\
\hline & Recessive & $\mathrm{GG} / \mathrm{GA}+\mathrm{AA}$ & $86 / 507$ & $74 / 489$ & $1.15(0.82-1.61)$ & $4.32 \mathrm{E}-01$ & $1.12(0.77-1.62)$ & 5.65E-01 \\
\hline \multirow[t]{3}{*}{ rs12026 } & Additive & $C>G$ & 192/1010 & $174 / 978$ & $1.09(0.86-1.37)$ & 4.80E-01 & $1.05(0.81-1.35)$ & 7.17E-01 \\
\hline & Dominant & $\mathrm{CC}+\mathrm{CG} / \mathrm{GG}$ & $173 / 505$ & $162 / 489$ & $1.05(0.81-1.37)$ & 7.07E-01 & $1.05(0.78-1.40)$ & $7.56 \mathrm{E}-01$ \\
\hline & Recessive & $\mathrm{CC} / \mathrm{CG}+\mathrm{GG}$ & $19 / 505$ & $12 / 489$ & $1.55(0.75-3.24)$ & $2.39 \mathrm{E}-01$ & $1.13(0.51-2.50)$ & $7.72 \mathrm{E}-01$ \\
\hline \multirow[t]{3}{*}{ rs 1053275} & Additive & $A>G$ & $203 / 1000$ & $186 / 994$ & $1.10(0.89-1.37)$ & $3.82 \mathrm{E}-01$ & $1.10(0.86-1.39)$ & 4.53E-01 \\
\hline & Dominant & $A A+A G / G G$ & $179 / 500$ & $165 / 497$ & $1.12(0.86-1.46)$ & $3.88 \mathrm{E}-01$ & $1.13(0.85-1.51)$ & $3.90 \mathrm{E}-01$ \\
\hline & Recessive & $\mathrm{AA} / \mathrm{AG}+\mathrm{GG}$ & $24 / 500$ & $21 / 497$ & $1.14(0.63-2.08)$ & $6.62 \mathrm{E}-01$ & $1.04(0.54-1.99)$ & $9.17 \mathrm{E}-01$ \\
\hline \multirow[t]{3}{*}{ rs705381 } & Additive & $G>A$ & $106 / 988$ & $151 / 990$ & $0.67(0.51-0.87)$ & $3.13 \mathrm{E}-03^{*}$ & $0.67(0.50-0.89)$ & $5.80 \mathrm{E}-03^{*}$ \\
\hline & Dominant & $\mathrm{GG}+\mathrm{GA} / \mathrm{AA}$ & $95 / 494$ & $144 / 495$ & $0.58(0.43-0.78)$ & $3.20 \mathrm{E}-04^{*}$ & $0.57(0.41-0.79)$ & $7.10 \mathrm{E}-04^{*}$ \\
\hline & Recessive & $\mathrm{GG} / \mathrm{GA}+\mathrm{AA}$ & $11 / 494$ & $7 / 495$ & $1.59(0.61-4.13)$ & $3.43 \mathrm{E}-01$ & $1.64(0.58-4.61)$ & $3.52 \mathrm{E}-01$ \\
\hline \multirow[t]{3}{*}{ rs2074353 } & Additive & $A>G$ & $253 / 996$ & $230 / 982$ & $1.11(0.91-1.36)$ & $3.16 \mathrm{E}-01$ & $1.12(0.90-1.40)$ & 3.09E-01 \\
\hline & Dominant & $A A+A G / G G$ & $219 / 498$ & $197 / 491$ & $1.17(0.91-1.51)$ & 2.20E-01 & $1.23(0.93-1.62)$ & $1.46 \mathrm{E}-01$ \\
\hline & Recessive & $\mathrm{AA} / \mathrm{AG}+\mathrm{GG}$ & $34 / 498$ & $33 / 491$ & $1.02(0.62-1.67)$ & $9.47 \mathrm{E}-01$ & $0.91(0.53-1.57)$ & 7.31E-01 \\
\hline \multirow[t]{3}{*}{ rs 854560} & Additive & $A>T$ & $41 / 1014$ & $39 / 996$ & 1.03(0.66-1.61) & 8.84E-01 & $0.95(0.58-1.56)$ & 8.37E-01 \\
\hline & Dominant & $A A+A T / T T$ & $40 / 507$ & $38 / 498$ & $1.04(0.65-1.65)$ & $8.78 \mathrm{E}-01$ & $0.97(0.58-1.62)$ & $9.12 \mathrm{E}-01$ \\
\hline & Recessive & $\mathrm{AA} / \mathrm{AT}+\mathrm{TT}$ & $1 / 507$ & $1 / 498$ & $0.98(0.06-15.75)$ & $9.90 \mathrm{E}-01$ & $0.40(0.02-7.40)$ & 5.39E-01 \\
\hline
\end{tabular}

Variants are described as minor allele or geno; the contrast allele refers to the minor allele; OR: odds ratio; $\mathrm{Cl}$ : confidence interval; $P_{\text {unjusted: }}$ unadjusted $\mathrm{P}$-value from t-test; $P_{\text {adjusted: }} \mathrm{P}$ value adjusted using logistic regression analysis with age, $\mathrm{HD}$ and hypertension as covariates. $\mathrm{F}_{-}$Stroke and $\mathrm{F} \_$Control represent the frequency of minor allele or geno in total stroke patients and controls respectively. Significant $\mathrm{P}$ values $(P<0.05)$ are in bold and $P^{*}<0.005($ Bonferroni multiple correction threshold).

association analysis was performed using logistic regression with or without adjustment for covariates. A single site association test between rs662 and rs854560 and ischemic stroke was conducted using an allele-based model. Bonferroni correction was undertaken for the 10 SNPs that were adopted into the single site association analysis.

Linkage disequilibrium analysis and haplotype selection were performed using Haploview software with parameter settings for pairwise tagging with $D^{\prime}>0.95$ [33]. The Omnibus ANOVA test was conducted using $\mathrm{R}$ software [34].

Inverse variance meta-analysis (RevMan 4.0 software) was used to contextualize our studies with two metaanalyses, using the data from PMID: 20856122 [35] and
PMID: 18511872 [30], which also studied the association between rs662 and rs854560 loci and ischemic stroke.

Values of $P<0.005$ were considered to represent the threshold for statistical significance.

\section{Results}

Clinical characteristics of total stroke patients and controls

Table 1 shows demographic characteristics and clinical vascular variables in the control and total stroke patients. There were no significant differences in levels of TC, TG and LDL between the controls and total stroke cases. However, HDL levels were significantly lower in stroke 
Table 3 Association between SNPs with ischemic stroke using the additive, dominant, genotype, and the recessive models

\begin{tabular}{|c|c|c|c|c|c|c|c|c|}
\hline \multirow[t]{2}{*}{ SNP } & \multirow[t]{2}{*}{ Model } & \multirow{2}{*}{$\begin{array}{l}\text { Allele or } \\
\text { geno }\end{array}$} & \multirow[t]{2}{*}{ F_IS } & \multirow[t]{2}{*}{ Control } & \multicolumn{2}{|l|}{ T-Statistic } & \multicolumn{2}{|c|}{ Logistic Regression } \\
\hline & & & & & OR $(95 \% \mathrm{Cl})$ & $\overline{P_{\text {unadjusted }}}$ & OR $(95 \% \mathrm{Cl})$ & $P_{\text {adjusted }}$ \\
\hline \multirow[t]{3}{*}{ rs854571 } & Additive & $C>T$ & $200 / 660$ & $344 / 982$ & $0.80(0.65-0.99)$ & 4.34E-02 & $0.84(0.66-1.07)$ & $1.62 \mathrm{E}-01$ \\
\hline & Dominant & $\mathrm{CC}+\mathrm{CT} / \mathrm{TT}$ & $170 / 330$ & $289 / 491$ & $0.74(0.56-0.98)$ & $3.79 \mathrm{E}-02$ & $0.80(0.58-1.10)$ & 1.63E-01 \\
\hline & Recessive & $\mathrm{CC} / \mathrm{CT}+\mathrm{TT}$ & $30 / 330$ & $55 / 491$ & $0.79(0.50-1.27)$ & $3.31 \mathrm{E}-01$ & $0.82(0.49-1.37)$ & 4.45E-01 \\
\hline \multirow[t]{3}{*}{ rs13306698 } & Additive & $A>G$ & $62 / 676$ & 98/988 & $0.91(0.65-1.29)$ & $6.00 \mathrm{E}-01$ & $1.07(0.72-1.59)$ & 7.43E-01 \\
\hline & Dominant & $A A+A G / G G$ & $61 / 338$ & $96 / 494$ & $0.91(0.64-1.30)$ & $6.16 \mathrm{E}-01$ & $1.09(0.72-1.64)$ & $6.83 \mathrm{E}-01$ \\
\hline & Recessive & $\mathrm{AA} / \mathrm{AG}+\mathrm{GG}$ & $1 / 338$ & $2 / 494$ & $0.73(0.07-8.08)$ & $7.98 \mathrm{E}-01$ & $0.55(0.04-7.85)$ & $6.63 \mathrm{E}-01$ \\
\hline \multirow[t]{3}{*}{ rs854572 } & Additive & $C>G$ & $285 / 668$ & $413 / 964$ & $0.99(0.82-1.21)$ & 9.44E-01 & $0.98(0.78-1.23)$ & $8.29 \mathrm{E}-01$ \\
\hline & Dominant & $C C+C G / G G$ & $220 / 334$ & $324 / 482$ & $0.94(0.70-1.27)$ & $6.87 \mathrm{E}-01$ & $0.94(0.67-1.32)$ & $7.23 \mathrm{E}-01$ \\
\hline & Recessive & $\mathrm{CC} / \mathrm{CG}+\mathrm{GG}$ & $65 / 334$ & $89 / 482$ & $1.07(0.75-1.52)$ & 7.21E-01 & $1.01(0.67-1.53)$ & $9.70 \mathrm{E}-01$ \\
\hline \multirow[t]{3}{*}{ rs7493 } & Additive & $C>G$ & $124 / 676$ & $176 / 974$ & $1.02(0.79-1.32)$ & $8.85 \mathrm{E}-01$ & $0.98(0.73-1.31)$ & $8.82 \mathrm{E}-01$ \\
\hline & Dominant & $C C+C G / G G$ & $114 / 338$ & $163 / 487$ & $1.01(0.75-1.36)$ & 9.39E-01 & $0.99(0.71-1.39)$ & $9.68 \mathrm{E}-01$ \\
\hline & Recessive & $\mathrm{CC} / \mathrm{CG}+\mathrm{GG}$ & $10 / 338$ & $13 / 487$ & $1.11(0.48-2.57)$ & $8.04 \mathrm{E}-01$ & $0.85(0.34-2.12)$ & $7.23 \mathrm{E}-01$ \\
\hline \multirow[t]{3}{*}{ rs662 } & Additive & $G>A$ & $276 / 674$ & $356 / 978$ & $1.19(0.98-1.45)$ & $7.34 \mathrm{E}-02$ & $1.18(0.94-1.47)$ & $1.46 \mathrm{E}-01$ \\
\hline & Dominant & $G G+G A / A A$ & $212 / 337$ & $282 / 489$ & $1.25(0.94-1.66)$ & $1.31 \mathrm{E}-01$ & $1.20(0.86-1.66)$ & $2.84 \mathrm{E}-01$ \\
\hline & Recessive & $\mathrm{GG} / \mathrm{GA}+\mathrm{AA}$ & $64 / 337$ & $74 / 489$ & $1.32(0.91-1.90)$ & $1.45 \mathrm{E}-01$ & $1.35(0.88-2.06)$ & $1.67 \mathrm{E}-01$ \\
\hline \multirow[t]{3}{*}{ rs12026 } & Additive & $C>G$ & $124 / 672$ & $174 / 978$ & $1.05(0.81-1.36)$ & 7.26E-01 & $1.02(0.76-1.37)$ & $8.78 \mathrm{E}-01$ \\
\hline & Dominant & $C C+C G / G G$ & $114 / 336$ & $162 / 389$ & 1.04(0.77-1.39) & $8.11 \mathrm{E}-01$ & $1.04(0.74-1.45)$ & $8.33 \mathrm{E}-01$ \\
\hline & Recessive & $\mathrm{CC} / \mathrm{CG}+\mathrm{GG}$ & $10 / 336$ & $12 / 489$ & $1.22(0.52-2.86)$ & $6.48 \mathrm{E}-01$ & $0.95(0.38-2.42)$ & $9.21 \mathrm{E}-01$ \\
\hline \multirow[t]{3}{*}{ rs 1053275} & Additive & $A>G$ & $144 / 666$ & 186/994 & $1.19(0.94-1.52)$ & $1.51 \mathrm{E}-01$ & $1.17(0.89-1.54)$ & 2.70E-01 \\
\hline & Dominant & $A A+A G / G G$ & $129 / 333$ & $165 / 497$ & $1.27(0.95-1.70)$ & $1.02 \mathrm{E}-01$ & $1.24(0.89-1.73)$ & $2.10 \mathrm{E}-01$ \\
\hline & Recessive & $\mathrm{AA} / \mathrm{AG}+\mathrm{GG}$ & $15 / 333$ & $21 / 497$ & $1.07(0.54-2.11)$ & $8.47 \mathrm{E}-01$ & $1.07(0.49-2.31)$ & $8.68 \mathrm{E}-01$ \\
\hline \multirow[t]{3}{*}{ rs705381 } & Additive & $G>A$ & $74 / 660$ & $151 / 990$ & $0.70(0.52-0.95)$ & $2.02 \mathrm{E}-02$ & $0.65(0.47-0.92)$ & $1.35 \mathrm{E}-02$ \\
\hline & Dominant & $G G+G A / A A$ & $65 / 330$ & $144 / 395$ & $0.60(0.43-0.83)$ & $2.50 \mathrm{E}-03^{*}$ & $0.54(0.37-0.79)$ & $1.67 \mathrm{E}-03^{*}$ \\
\hline & Recessive & $G G / G A+A A$ & $9 / 330$ & $7 / 495$ & $1.96(0.72-5.30)$ & $1.88 \mathrm{E}-01$ & $1.85(0.60-5.64)$ & $2.83 \mathrm{E}-01$ \\
\hline \multirow[t]{3}{*}{ rs2074353 } & Additive & $A>G$ & $176 / 662$ & $230 / 982$ & $1.18(0.94-1.47)$ & $1.53 \mathrm{E}-01$ & $1.23(0.95-1.60)$ & 1.09E-01 \\
\hline & Dominant & $A A+A G / G G$ & $153 / 331$ & $197 / 491$ & $1.28(0.97-1.70)$ & $8.30 \mathrm{E}-02$ & $1.38(1.00-1.91)$ & 5.29E-02 \\
\hline & Recessive & $\mathrm{AA} / \mathrm{AG}+\mathrm{GG}$ & $23 / 331$ & $33 / 491$ & $1.04(0.60-1.80)$ & $8.99 \mathrm{E}-01$ & $1.06(0.56-1.99)$ & $8.69 \mathrm{E}-01$ \\
\hline \multirow[t]{3}{*}{ rs854560 } & Additive & $A>T$ & $30 / 674$ & $39 / 996$ & $1.14(0.70-1.85)$ & 5.93E-01 & $1.19(0.69-2.07)$ & $5.36 \mathrm{E}-01$ \\
\hline & Dominant & $A A+A T / T T$ & $29 / 337$ & $38 / 498$ & $1.14(0.69-1.89)$ & $6.11 \mathrm{E}-01$ & $1.24(0.70-2.21)$ & $4.57 \mathrm{E}-01$ \\
\hline & Recessive & $\mathrm{AA} / \mathrm{AT}+\mathrm{TT}$ & $1 / 337$ & $1 / 498$ & $1.48(0.09-23.73)$ & 7.82E-01 & $0.43(0.02-9.62)$ & $5.92 \mathrm{E}-01$ \\
\hline
\end{tabular}

Variants are described as minor allele or geno and the contrast allele refers to the minor allele; $O R$ : odds ratio; $\mathrm{Cl}$ : confidence interval; $P_{\text {unjusted: }}$ unadjusted $\mathrm{P}$-value from t-test; $P_{\text {adjusted: }}$ P value adjusted using logistic regression analysis with age, HD and hypertension as covariates $F \_I S$ and $F \_C o n t r o l$ represent the frequency of minor allele in ischemic stroke patients and controls respectively. Significant $\mathrm{P}$ values $(P<0.05)$ are in bold and $P^{*}<0.005$ (Bonferroni multiple correction threshold).

cases than in controls and mean age and incidence of hypertension were significantly higher.

\section{Linkage disequilibrium}

A total of eleven gene polymorphisms were genotyped in the cases and controls. For PON1 these included three coding-region polymorphisms (rs662/Q192R, rs13306698/ Arg160Gly, and rs854560/Leu55Met) and four regulatoryregion polymorphisms (rs705379/-107/-108, rs705381/160/-162, rs854571/-824/-832, and rs854572/-907/-909). There were also two coding-region polymorphisms of PON2 (rs12026/Ala148Gly, and rs7493/Ser311Cys), and two coding-region polymorphisms of PON3 (rs2074353 located in intron and rs1053275/Ala99Ala). The total rate of successful genotyping was $98.6 \%$. All genotype distributions within the studied polymorphisms were in HardyWeinberg equilibrium $(P>0.05)$, in both cases and controls, except for rs705379 $(-107 /-108)(P<0.001)$, which was located in the promoter of PON1.

The results of linkage disequilibrium evaluation analyses are shown in Figure 1A. In this analysis, SNPs with a pairwise $r^{2}>0.9$ were considered to be in the same block. Based on this approach, four haplotype blocks (Block1: rs854560-rs13306698-rs662; Block2: rs854572rs854571-rs705381; Block3: rs1053275-rs2074353; Block4: rs12026-rs7493) were identified (Figure 1B). 
Table 4 Association between SNPs and hemorrhagic stroke using the additive, dominant, genotype, and the recessive models

\begin{tabular}{|c|c|c|c|c|c|c|c|c|}
\hline \multirow[t]{2}{*}{ SNP } & \multirow[t]{2}{*}{ Model } & \multirow{2}{*}{$\begin{array}{l}\text { Allele or } \\
\text { geno }\end{array}$} & \multirow[t]{2}{*}{ F_HS } & \multirow[t]{2}{*}{ F_Control } & \multicolumn{2}{|l|}{ T-Statistic } & \multicolumn{2}{|c|}{ Logistic Regression } \\
\hline & & & & & OR $(95 \% \mathrm{Cl})$ & $P_{\text {unadjusted }}$ & OR $(95 \% \mathrm{Cl})$ & $P_{\text {adjusted }}$ \\
\hline \multirow[t]{3}{*}{ rs854571 } & Additive & $C>T$ & $92 / 316$ & $344 / 982$ & $0.76(0.57-1.00)$ & $5.00 \mathrm{E}-02$ & $0.76(0.57-1.01)$ & $5.54 \mathrm{E}-02$ \\
\hline & Dominant & $\mathrm{CC}+\mathrm{CT} / \mathrm{TT}$ & $78 / 158$ & $289 / 491$ & $0.68(0.48-0.98)$ & $3.68 \mathrm{E}-02$ & $0.70(0.48-1.01)$ & 5.57E-02 \\
\hline & Recessive & $\mathrm{CC} / \mathrm{CT}+\mathrm{TT}$ & $14 / 158$ & $55 / 491$ & $0.77(0.42-1.43)$ & $4.08 \mathrm{E}-01$ & $0.71(0.38-1.34)$ & 2.95E-01 \\
\hline \multirow[t]{3}{*}{ rs13306698 } & Additive & $A>G$ & $33 / 324$ & $98 / 988$ & $1.03(0.67-1.59)$ & 8.85E-01 & 1.06(0.68-1.66) & 7.93E-01 \\
\hline & Dominant & $A A+A G / G G$ & $33 / 162$ & $96 / 494$ & $1.06(0.68-1.65)$ & 7.95E-01 & $1.09(0.69-1.72)$ & 7.06E-01 \\
\hline & Recessive & $\mathrm{AA} / \mathrm{AG}+\mathrm{GG}$ & $0 / 162$ & $2 / 494$ & 0.00 (0.00-inf) & $9.99 \mathrm{E}-01$ & 0.00 (0.00-inf) & $9.99 \mathrm{E}-01$ \\
\hline \multirow[t]{3}{*}{ rs854572 } & Additive & $C>G$ & $151 / 320$ & $413 / 964$ & $1.20(0.93-1.54)$ & $1.73 \mathrm{E}-01$ & $1.24(0.95-1.61)$ & $1.20 \mathrm{E}-01$ \\
\hline & Dominant & $\mathrm{CC}+\mathrm{CG} / \mathrm{GG}$ & $118 / 160$ & $324 / 482$ & $1.37(0.92-2.04)$ & 1.23E-01 & $1.38(0.91-2.08)$ & 1.27E-01 \\
\hline & Recessive & $\mathrm{CC} / \mathrm{CG}+\mathrm{GG}$ & $33 / 160$ & $89 / 482$ & $1.15(0.73-1.79)$ & $5.46 \mathrm{E}-01$ & $1.25(0.79-1.99)$ & $3.38 \mathrm{E}-01$ \\
\hline \multirow[t]{3}{*}{ rs7493 } & Additive & $C>G$ & $64 / 324$ & $176 / 974$ & $1.12(0.81-1.55)$ & 4.94E-01 & $1.05(0.75-1.46)$ & 7.77E-01 \\
\hline & Dominant & $\mathrm{CC}+\mathrm{CG} / \mathrm{GG}$ & $57 / 162$ & $163 / 487$ & 1.08(0.74-1.57) & $6.90 \mathrm{E}-01$ & $1.03(0.70-1.51)$ & $8.94 \mathrm{E}-01$ \\
\hline & Recessive & $\mathrm{CC} / \mathrm{CG}+\mathrm{GG}$ & $7 / 162$ & $13 / 487$ & $1.65(0.65-4.20)$ & $2.97 \mathrm{E}-01$ & $1.28(0.49-3.36)$ & $6.13 \mathrm{E}-01$ \\
\hline \multirow[t]{3}{*}{ rs662 } & Additive & $G>A$ & $108 / 324$ & $356 / 978$ & $0.88(0.68-1.14)$ & $3.36 \mathrm{E}-01$ & $0.85(0.65-1.12)$ & $2.48 \mathrm{E}-01$ \\
\hline & Dominant & $\mathrm{GG}+\mathrm{GA} / \mathrm{AA}$ & $88 / 162$ & $282 / 489$ & $0.87(0.61-1.25)$ & 4.56E-01 & $0.82(0.56-1.18)$ & 2.85E-01 \\
\hline & Recessive & $\mathrm{GG} / \mathrm{GA}+\mathrm{AA}$ & 20/162 & $74 / 489$ & $0.79(0.47-1.34)$ & 3.83E-01 & $0.80(0.46-1.38)$ & 4.26E-01 \\
\hline \multirow[t]{3}{*}{ rs 12026} & Additive & $C>G$ & $64 / 322$ & $174 / 978$ & $1.15(0.83-1.59)$ & 3.95E-01 & $1.10(0.78-1.53)$ & $5.92 \mathrm{E}-01$ \\
\hline & Dominant & $\mathrm{CC}+\mathrm{CG} / \mathrm{GG}$ & $57 / 161$ & $162 / 389$ & $1.11(0.76-1.61)$ & 5.96E-01 & $1.07(0.72-1.57)$ & 7.44E-01 \\
\hline & Recessive & $\mathrm{CC} / \mathrm{CG}+\mathrm{GG}$ & $7 / 161$ & $12 / 489$ & $1.81(0.70-4.67)$ & $2.22 \mathrm{E}-01$ & $1.47(0.55-3.92)$ & 4.39E-01 \\
\hline \multirow[t]{3}{*}{ rs 1053275} & Additive & $A>G$ & $58 / 318$ & $186 / 994$ & $0.97(0.71-1.33)$ & $8.55 \mathrm{E}-01$ & $0.96(0.70-1.33)$ & 8.07E-01 \\
\hline & Dominant & $\mathrm{AA}+\mathrm{AG} / \mathrm{GG}$ & $49 / 159$ & $165 / 497$ & $0.90(0.61-1.32)$ & 5.77E-01 & $0.91(0.61-1.35)$ & $6.36 \mathrm{E}-01$ \\
\hline & Recessive & $\mathrm{AA} / \mathrm{AG}+\mathrm{GG}$ & $9 / 159$ & $21 / 497$ & $1.36(0.61-3.03)$ & 4.53E-01 & $1.17(0.51-2.69)$ & 7.09E-01 \\
\hline \multirow[t]{3}{*}{ rs705381 } & Additive & $G>A$ & $32 / 312$ & $151 / 990$ & $0.62(0.41-0.94)$ & $2.42 \mathrm{E}-02$ & $0.62(0.40-0.95)$ & 2.90E-02 \\
\hline & Dominant & $\mathrm{GG}+\mathrm{GA} / \mathrm{AA}$ & $30 / 156$ & $144 / 395$ & $0.58(0.37-0.90)$ & $1.61 \mathrm{E}-02$ & $0.57(0.36-0.90)$ & $1.65 \mathrm{E}-02$ \\
\hline & Recessive & $\mathrm{GG} / \mathrm{GA}+\mathrm{AA}$ & $2 / 156$ & $7 / 495$ & $0.91(0.19-4.40)$ & $9.02 \mathrm{E}-01$ & $1.22(0.24-6.22)$ & 8.13E-01 \\
\hline \multirow[t]{3}{*}{ rs2074353 } & Additive & $A>G$ & $74 / 340$ & 230/982 & $0.99(0.74-1.32)$ & 9.57E-01 & $0.96(0.72-1.29)$ & 8.05E-01 \\
\hline & Dominant & $\mathrm{AA}+\mathrm{AG} / \mathrm{GG}$ & $63 / 159$ & $197 / 491$ & $0.98(0.68-1.41)$ & $9.11 \mathrm{E}-01$ & $0.98(0.67-1.42)$ & $8.98 \mathrm{E}-01$ \\
\hline & Recessive & $\mathrm{AA} / \mathrm{AG}+\mathrm{GG}$ & $11 / 159$ & $33 / 491$ & $1.03(0.51-2.09)$ & $9.31 \mathrm{E}-01$ & $0.87(0.42-1.82)$ & 7.21E-01 \\
\hline \multirow[t]{3}{*}{ rs854560 } & Additive & $A>T$ & $9 / 324$ & $39 / 996$ & $0.70(0.34-1.46)$ & 3.46E-01 & $0.57(0.26-1.25)$ & $1.58 \mathrm{E}-01$ \\
\hline & Dominant & $\mathrm{AA}+\mathrm{AT} / \mathrm{TT}$ & 9/162 & $38 / 498$ & $0.71(0.34-1.51)$ & $3.74 \mathrm{E}-01$ & $0.57(0.26-1.28)$ & $1.74 \mathrm{E}-01$ \\
\hline & Recessive & $\mathrm{AA} / \mathrm{AT}+\mathrm{T}$ & $0 / 162$ & NA & NA & NA & NA & NA \\
\hline
\end{tabular}

Variants are described as minor allele or geno and the contrast allele refers to the minor allele; $P_{\text {unjusted: }}$ unadjusted P-value from t-test; $P_{\text {adjusted: }} \mathrm{P}$ value adjusted using logistic regression analysis with age, HD and hypertension as covariates. F_HS and F_Control represent the frequency of minor allele in hemorrhagic stroke patients and controls respectively. NA means not applicable. Significant $P$ values $(P<0.05)$ are in bold.

\section{Single site association}

The association between the ten SNPs included in the four blocks and total stroke occurrence was analyzed using additive, dominant, genotype, and recessive models. As shown in Table 2, two polymorphisms, rs705381 and rs854571 were significantly associated with total stoke using additive and dominant models. The allele A of rs705381 and the allele $\mathrm{T}$ of rs854571 were both less frequent in patients with total stroke than in controls. The association remained significant after logistic regression analysis adjusting for age, HDL and hypertension using the additive model (rs705381, $P_{\text {adjusted }}=0.0058, O R=0.67$ $[95 \% \mathrm{CI}=0.50-0.89]$; and $\mathrm{rs} 854571, P_{\text {adjusted }}=0.0330, \mathrm{OR}$
$=0.80[95 \% \mathrm{CI}=0.65-0.98])$. However, both P-values failed to reach significance after the Bonferroni adjustment for multiple comparisons. Analysis using the dominant model, showed that the differences in rs705381 remained significant after Bonferroni correction $\left(P_{\text {adjusted }}=0.0007\right.$, $O R=0.57[95 \% \mathrm{CI}=0.41-0.79]$ ), but the differences in rs854571 did not. There was no significant association between any of the SNPs of PON genes and total strokes when analyzed using the recessive model.

As shown in Table 3, rs705381 was significantly associated with ischemic stroke after adjustment of confounders in both additive and dominant models $\left(P_{\text {adjusted }}=0.0017, \quad\right.$ OR $\left.=0.54 \quad[95 \% \quad \mathrm{CI}=0.37-0.79]\right)$. 
Table 5 Haplotypes of the four blocks between total strokes and control subjects

\begin{tabular}{|c|c|c|c|c|}
\hline \multirow[t]{2}{*}{ Haplotype } & \multicolumn{4}{|c|}{ Logistic Regression } \\
\hline & OR & $P_{\text {unadjusted }}$ & OR & $P_{\text {adjusted }}$ \\
\hline \multicolumn{5}{|c|}{ Block1: rs854560-rs13306698-rs662 } \\
\hline OMNIBUS & NA & 0.9371 & NA & 0.9569 \\
\hline TAA & 1.03 & 0.8820 & 0.95 & 0.8390 \\
\hline AAA & 1.08 & 0.4170 & 1.06 & 0.5790 \\
\hline AGG & 0.95 & 0.7640 & 0.98 & 0.8840 \\
\hline$\overline{\mathrm{AAG}}$ & 0.94 & 0.4810 & 0.96 & 0.6580 \\
\hline
\end{tabular}

Block2: rs854572-rs854571-rs705381

\begin{tabular}{lllll}
\hline OMNIBUS & NA & $\mathbf{0 . 0 1 2 9}$ & NA & $\mathbf{0 . 0 3 9 4}$ \\
\hline CTT & 1.05 & 0.6170 & 1.08 & 0.4200 \\
\hline CTC & 0.64 & $\mathbf{0 . 0 0 1 3}$ & 0.65 & $\mathbf{0 . 0 0 3 7}$ \\
\hline GCC & 0.99 & 0.9110 & 0.99 & 0.9280 \\
\hline CCC & 1.24 & $\mathbf{0 . 0 4 4 2}$ & 1.19 & 0.1420
\end{tabular}

Block3: rs 1053275-rs2074353

\begin{tabular}{lllll}
\hline OMNIBUS & NA & 0.4970 & NA & 0.5757 \\
\hline GG & 1.10 & 0.3970 & 1.10 & 0.4210 \\
\hline AG & 1.09 & 0.6630 & 1.13 & 0.5880 \\
\hline AA & 0.90 & 0.2920 & 0.89 & 0.3010 \\
\hline
\end{tabular}

Block4: rs12026-rs7493

\begin{tabular}{lllll}
\hline OMNIBUS & NA & 0.2479 & NA & 0.5467 \\
\hline GG & 1.08 & 0.5390 & 1.03 & 0.8430 \\
\hline CC & 0.92 & 0.5020 & 0.96 & 0.7660 \\
\hline
\end{tabular}

Haplotypes observed in $<1 \%$ of the control subjects are not listed in the table. $O R$ : odds ratio; $P_{\text {unjusted: }}$ unadjusted P-value from t-test; $P_{\text {adjusted: }} \mathrm{P}$ value adjusted using logistic regression analysis with age, HD and hypertension as covariates. OMIBUS value was calculated by an ANOVA analysis for including or not including the haplotype information in a likelihood ration test of nested model. The OR in one block for each haplotype was calculated by using all the other haplotypes in the same block as the reference haplotype. Significant $P$ values $(P<0.05)$ are in bold.

However, no significant association with ischemic stroke was found using the recessive model.

Rs854571 was associated with hemorrhagic stroke, with marginal significance ( $P_{\text {unadjusted }}=0.0500, O R=0.76[95 \%$ $\mathrm{CI}=0.57-1.00])$ using the additive model, and rs705381 showed a significant association in both additive $\left(P_{\text {adjusted }}=0.0290, O R=0.62[95 \% \mathrm{CI}=0.40-0.95]\right)$ and dominant models ( $P_{\text {adjusted }}=0.0165, O R=0.57[95 \%$ $\mathrm{CI}=0.36-0.90])$ (Table 4). However, neither of the two SNPs was significantly associated with hemorrhagic stroke after the Bonferroni correction. Thus, there was no significant finding for hemorrhagic stroke with any of the three models.

\section{Haplotype analysis}

Haplotype analysis conducted in the four blocks, with or without adjustment for age, HDL and hypertension as covariates is shown in Table 5. Block 2 consisting of rs854572, rs854571 and rs705381 was associated with total stroke $(P=0.0129$ Omnibus test), and included one protective haplotype $\quad \mathrm{C}-\mathrm{T}-\mathrm{C} \quad\left(O R=0.64 ; \quad P_{\text {unadjusted }}=0.0013,\right)$ and one nominal risk haplotype $\mathrm{C}-\mathrm{C}-\mathrm{C}(\mathrm{OR}=1.24$; $P_{\text {unadjusted }}=0.0442$, ). The association for haplotype C$\mathrm{T}-\mathrm{C}$ remained significant after adjustment for age, HDL and hypertension as covariates $(O R=0.65 ; P=0.0037)$. No other significant haplotype associations were found.

\section{Meta-analysis}

Two meta-analyses, PMID: 20856122 [35] and PMID: 18511872 [30], which studied the association between rs662 and rs854560 loci and ischemic stroke were contextualized with our study using the random effects model. Forests plot for rs662 from 25 studies including our own are shown in Figure 2. There was a nominal significant association between rs662 and ischemic stroke $(P=0.0100, O R=1.08[95 \% \mathrm{CI}=1.02-1.15])$ yielding 1.08 per $\mathrm{G}$ allele copy, with no statistical evidence for statistical heterogeneity $\left(P=0.0400, I^{2}=36 \%\right)$ between studies.

There was no evidence of an association between rs854560 and ischemic stroke risk $(P=0.3700, O R=0.97$ [95\% CI $=0.91-1.04])$ and no evidence of heterogeneity $\left(P=0.2700, I^{2}=16 \%\right)$ between studies (Figure 3$)$.

\section{Discussion}

The present study investigated the association of 11 polymorphisms in $3 P O N$ genes with the risk of stroke. Using a dominant model, we demonstrated that rs705381 $(-162)$ was significantly associated with total stroke and ischemic stroke but not with hemorrhagic stroke. There was also a nominal association between rs854571 (-824) and stroke with the allele $\mathrm{T}$ as a protective factor.

Both rs705381 and rs854571 polymorphisms located in the promoter region of PON1 were associated with stroke, which was consistent with previous findings [19,36-39]. The protective effect of $-162 \mathrm{~T}$ polymorphism on total stroke and ischemic stroke was also consistent with previous observations [40] which suggested that NF-1, a ubiquitous nuclear factor and a transcriptional activator, has a binding site on PON1 if allele A appears at -162 . Other studies have shown that $-162 \mathrm{~T}$ polymorphism results in higher expression levels of PON1 [40,41] There is also evidence to suggest a correlation between AA $(-162)$ and high PON activities in Caucasians [42].

Our results support the hypothesis that the protective effect of $-162 \mathrm{~T}$ polymorphism might be attributable to high PON activity [42]. We also found weak evidence to suggest that $-824 \mathrm{~T}$ was associated with a reduced propensity to suffer stroke. However, the evidence was no longer apparent after Bonferroni correction for multiple comparisons. It has been previously reported that $-824 \mathrm{~T}$ (824A in their finding) was associated with low serum PON levels [43]. Negative associations between -162 and -824 have been reported in studies in American populations $[23,40]$. These findings highlight the potential influence of 


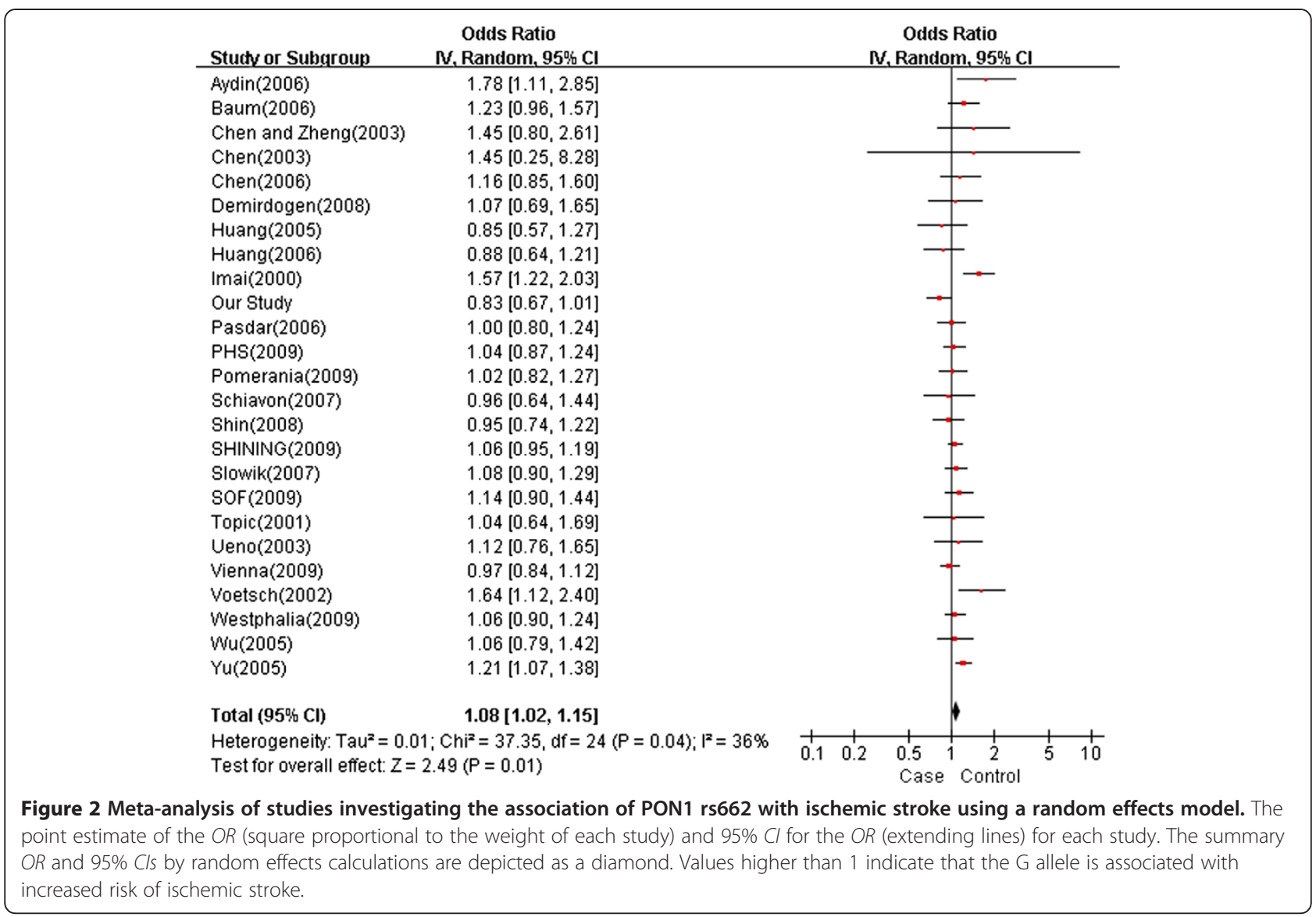

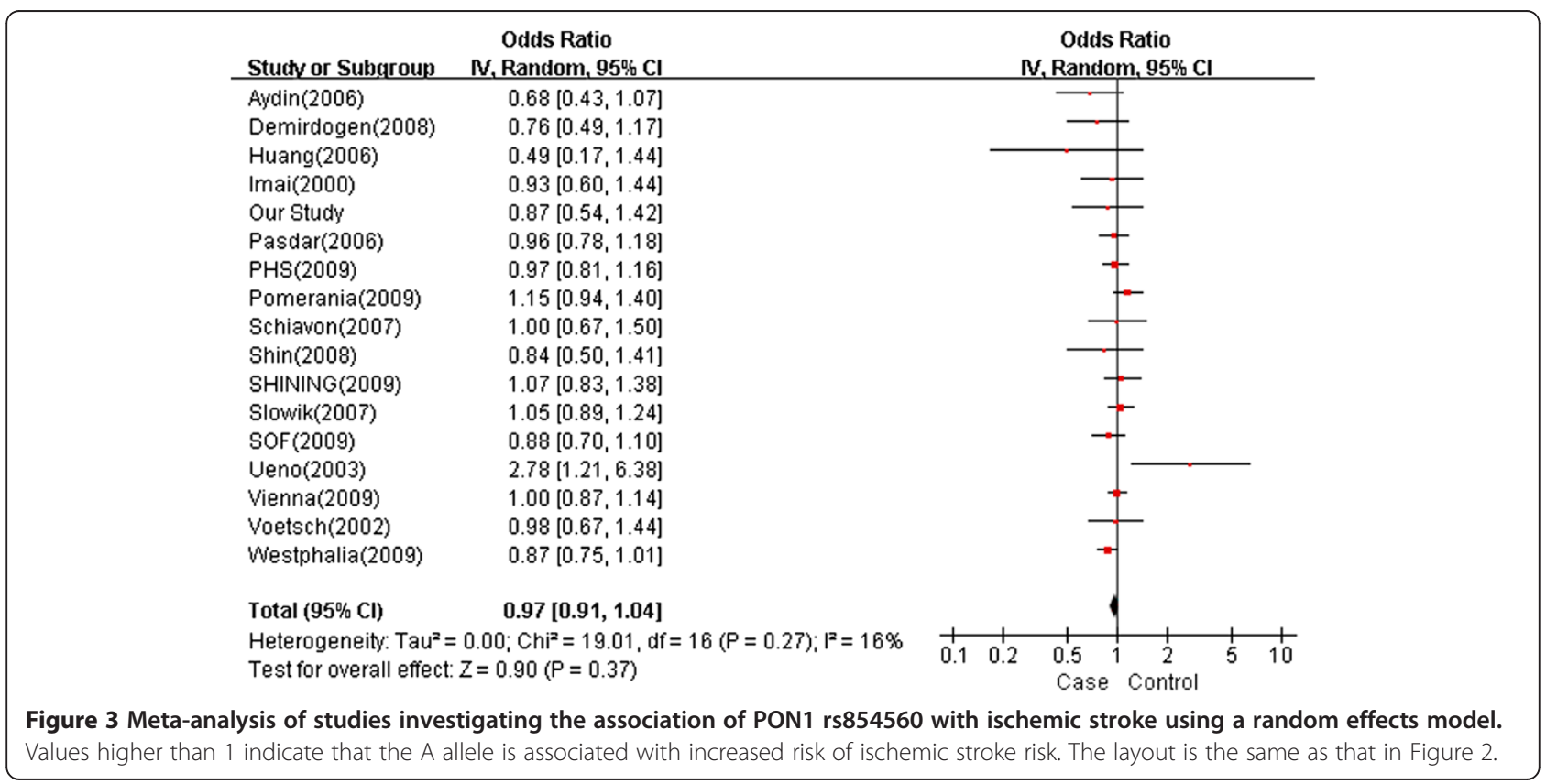


ethnic differences in terms of the founder effect and identical-by-descent principles $[44,45]$.

Patients with coronary heart disease (CHD) have been shown to have a higher frequency of $-162 \mathrm{~T}$ allele than the controls, suggesting allele A may be associated with risk of CHD in the Han Chinese population [46]. However, in our study, we found a protective effect of the $-162 \mathrm{~T}$ polymorphism on stroke.

Haplotype analysis further confirmed our positive results and identified a positive association between the protective haplotype C-T-C and the risk haplotype C-C-C of rs854572-rs854571-rs705381 (Block 2) with total stroke. No significant associations were observed for stroke susceptibility with the two coding region polymorphisms in PON2, which was consistent with previous findings in the Han Chinese population and in North Americans [24,29], although a positive association of Ser311Cys was found in a Polish population [22].

The absence of any positive correlations between stroke risk and the two PON3 polymorphisms in our study was also consistent with reported findings in Caucasian and North American patients [24,27].

Our study was conducted in a relatively large Chinese sample pool and included careful assessment of two stroke subtypes. We also selected common variants in all three members of the PON gene family. However, functional detection of PON activities was not undertaken in the present study and investigation of the association between SNPs and large or small vessel strokes was not possible as a complete classification of the subtype of ischemic stroke subjects was not available in our study. In our study, results from both adjusted and unadjusted analyses were in line with each other. However, in other settings, authorities have discouraged the use of data adjustments for the determination of the total genetic effect [47]. It, therefore, remains uncertain as to whether adjusted or unadjusted data should be used to interpret our results in clinical context.

\section{Conclusion}

The study identified rs705381 (-162) as being significantly associated with total stroke and ischemic stroke, and demonstrated a weak association for rs854571 (-824) in the Han Chinese population. These findings support the involvement of $P O N$ polymorphisms in the development of stroke. Further studies with larger sample sizes are required to validate these findings and to elucidate the underlying biological mechanisms.

\section{Competing interests}

The authors have no competing interests.

\section{Authors' contributions}

YS and YS designed the study, coordinated sample recruitment and revised the final manuscript. GZ participated in study design and collected the samples. WL drafted the manuscript and carried out the statistical analysis.
ZL helped with the statistical analysis and draft manuscript preparation. HL, $\mathrm{RM}$ and $\mathrm{XK}$ helped with the sample collection. YR and XL performed the SNP genotyping. All authors read and approved the final manuscript.

\section{Acknowledgements}

The project was supported by National Key Technology R\&D Program in the 11 th Five year Plan of China (2008BAI52B03), the Natural Science Foundation of China (81130022, 81272302, 31000553), the National 863 project (2012AA02A515), the Foundation for the Author of National Excellent Doctoral Dissertation of China (201026), and Shanghai Science and Technology Development Funds (12QA1401900).

\section{Author details}

${ }^{1}$ Laboratory Diagnosis Center, Beijing Tiantan Hospital Affiliated to Capital Medical University, Beijing 100050, China. ${ }^{2}$ Bio-X Institutes, Key Laboratory for the Genetics of Developmental and Neuropsychiatric Disorders (Ministry of Education), Shanghai Jiao Tong University, Shanghai 200030, China. ${ }^{3}$ CapitalBio Corporation, 18 Life Science Parkway, Changping District, Beijing 102206, China. ${ }^{4}$ National Engineering Research Center for Beijing Biochip Technology, 18 Life Science Parkway, Changping District, Beijing 102206, China.

Received: 3 April 2012 Accepted: 22 January 2013

Published: 28 January 2013

\section{References}

1. Adams H, Bendixen B, Kappelle L, Biller J, Love B, Gordon D, Marsh E: Classification of subtype of acute ischemic stroke. Definitions for use in a multicenter clinical trial. TOAST. Trial of Org 10172 in Acute Stroke Treatment. Stroke 1993, 24(1):35-41.

2. Liao DP, Myers R, Hunt S, Shahar E, Paton C, Burke G, Province M, Heiss G: Familial history of stroke and stroke risk - The family heart study. Stroke 1997, 28(10):1908-1912.

3. de Lange M, Snieder H, Ariens RAS, Spector TD, Grant PJ: The genetics of haemostasis: a twin study. Lancet 2001, 357(9250):101-105.

4. Bak S, Gaist D, Sindrup SH, Skytthe A, Christensen K: Genetic liability in stroke - a long-term follow-up study of Danish twins. Stroke 2002, 33(3):769-774

5. Rubattu S, Volpe M, Kreutz R, Ganten U, Ganten D, Lindpaintner K: Chromosomal mapping of quantitative trait loci contributing to stroke in a rat model of complex human disease. Nat Genet 1996, 13(4):429-434.

6. Jeffs B, Clark JS, Anderson NH, Gratton J, Brosnan MJ, Gauguier D, Reid JL, Macrae IM, Dominiczak AF: Sensitivity to cerebral ischaemic insult in a rat model of stroke is determined by a single genetic locus. Nat Genet 1997, 16(4):364-367.

7. Joutel A, Corpechot C, Ducros A, Vahedi K, Chabriat H, Mouton P, Alamowitch S, Domenga V, Cecillion M, Marechal E, et al: Notch3 mutations in CADASIL, a hereditary adult-onset condition causing stroke and dementia. Nature 1996, 383(6602):707-710.

8. Petersen NH, Schmied AB, Zeller JA, Plendl H, Deuschl G, Zunker P: Lp(a) lipoprotein and plasminogen activity in patients with different etiology of ischemic stroke. Cerebrovasc Dis 2007, 23(2-3):188-193.

9. Lernfelt B, Forsberg M, Blomstrand C, Mellstrom D, Volkmann R: Cerebral atherosclerosis as predictor of stroke and mortality in representative elderly population. Stroke 2002, 33(1):224-229.

10. Nagai $Y$, Kitagawa $K$, Sakaguchi M, Shimizu $Y$, Hashimoto $H$, Yamagami $H$, Narita M, Ohtsuki T, Hori M, Matsumoto M: Significance of earlier carotid atherosclerosis for stroke subtypes. Stroke 2001, 32(8):1780-1785.

11. Gordon DJ, Rifkind BM: High-density lipoprotein — the clinical implications of recent studies. N Eng J Med 1989, 321(19):1311-1316.

12. Kozarsky KF, Donahee MH, Glick JM, Krieger M, Rader DJ: Gene transfer and hepatic overexpression of the HDL receptor SR-BI reduces atherosclerosis in the cholesterol-fed LDL receptor-deficient mouse. Arterioscler Thromb Vasc Biol 2000, 20(3):721-727.

13. Primo-Parmo SL, Sorenson RC, Teiber J, Du BNL: The human serum paraoxonase/arylesterase gene (PON1) is one member of a multigene family. Genomics 1996, 33(3):498-507.

14. Reddy ST, Wadleigh DJ, Grijalva V, Ng C, Hama S, Gangopadhyay A, Shih DM, Lusis AJ, Navab M, Fogelman AM: Human paraoxonase-3 is an HDLassociated enzyme with biological activity similar to paraoxonase-1 
protein but is not regulated by oxidized lipids. Arterioscler Thromb VasC Biol 2001, 21(4):542-547.

15. Mackness Ml, Arrol S, Durrington PN: Paraoxonase prevents accumulation of lipoperoxides in low-density lipoprotein. FEBS Lett 1991, 286(1-2):152-154.

16. Aviram M, Rosenblat M, Bisgaier CL, Newton RS, Primo-Parmo SL, La Du BN: Paraoxonase inhibits high-density lipoprotein oxidation and preserves its functions - a possible peroxidative role for paraoxonase. J Clin Invest 1998, 101(8):1581-1590.

17. Tward A, Xia Y-R, Wang X-P, Shi Y-S, Park C, Castellani LW, Lusis AJ, Shih DM: Decreased atherosclerotic lesion formation in human serum paraoxonase transgenic mice. Circulation 2002, 106(4):484-490.

18. Mackness Ml, Arrol S, Abbott CA, Durrington PN: Is paraoxonase related to atherosclerosis. Chem Biol Interact 1993, 87(1-3):161-171.

19. Kim NS, Kang K, Cha MH, Kang BJ, Moon J, Kang BK, Yu BC, Kim YS, Choi SM, Bang OS: Decreased paraoxonase-1 activity is a risk factor for ischemic stroke in Koreans. Biochem Biophys Res Commun 2007, 364(1):157-162.

20. Michalak S, Kazmierski R, Hellmann A, Wysocka E, KocialkowskaAdamczewska D, Wencel-Warot A, Nowinski WL: Serum paraoxonase/ arylesterase activity affects outcome in ischemic stroke patients. Cerebrovasc Dis 2011, 32(2):124-132.

21. Martinelli N, Micaglio R, Consoli L, Guarini P, Grison E, Pizzolo F, Friso S, Trabetti E, Pignatti PF, Corrocher R, et al: Low levels of serum paraoxonase activities are characteristic of metabolic syndrome and may influence the metabolic-syndrome-related risk of coronary artery disease. Exp Diabetes Res 2012, 2012:231502.

22. Slowik A, Wloch D, Szermer P, Wolkow P, Malecki M, Pera J, Turaj W, Dziedzic T, Klimkowicz-Mrowiec A, Kopec G, et al: Paraoxonase 2 gene C311S polymorphism is associated with a risk of large vessel disease stroke in a Polish population. Cerebrovasc Dis 2007, 23(5-6):395-400.

23. Voetsch B, Benke KS, Panhuysen Cl, Damasceno BP, Loscalzo J: The combined effect of paraoxonase promoter and coding region polymorphisms on the risk of arterial ischemic stroke among young adults. Arch Neurol 2004, 61(3):351-356.

24. Ranade K, Kirchgessner TG, lakoubova OA, Devlin JJ, DelMonte T, Vishnupad P, Hui L, Tsuchihashi Z, Sacks FM, Sabatine MS, et al: Evaluation of the paraoxonases as candidate genes for stroke - GIn192Arg polymorphism in the paraoxonase 1 gene is associated with increased risk of stroke. Stroke 2005, 36(11):2346-2350.

25. Voetsch B, Benke KS, Damasceno BP, Siqueira LH, Loscalzo J: Paraoxonase 192 Gln - > Arg polymorphism - an independent risk factor for nonfatal arterial ischemic stroke among young adults. Stroke 2002, 33(6):1459-1464.

26. Xiao ZJ, Chen J, Sun Y, Zheng ZJ: Lack of association between the Paraoxonase $1 \mathrm{Q} / \mathrm{R} 192$ single nucleotide polymorphism and stroke in a Chinese cohort. Acta Neurol Belg 2009, 109(3):205-209.

27. Pasdar A, Ross-Adams H, Cumming A, Cheung J, Whalley L, St Clair D, MacLeod MJ: Paraoxonase gene polymorphisms and haplotype analysis in a stroke population. BMC Med Genet 2006, 7:28

28. Huang Q, Liu Y-h, Yang Q-d, Xiao B, Ge L, Zhang N, Xia J, Zhang L, Liu Z-j: Human serum paraoxonase gene polymorphisms, Q192R and L55M, are not associated with the risk of cerebral infarction in Chinese Han population. Neurolog Res 2006, 28(5):549-554.

29. Xu HW, Yuan N, Zhao Z, Zhang L, Xia J, Zeng KM, Xiao B, Yang XS, Tang BS: Study of the relationship between gene polymorphisms of paraoxonase 2 and stroke in a chinese population. Cerebrovasc Dis 2008, 25(1-2):87-94.

30. Xu XW, Li JJ, Sheng WL, Liu L: Meta-analysis of genetic studies from journals published in China of ischemic stroke in the Han Chinese population. Cerebrovasc Dis 2008, 26(1):48-62

31. Afridi I, Canny J, Yao CH, Christensen B, Cooper RS, Kadiri S, Hill S, Kaplan N, Kuschnir E, Lexchin J, et al: World Health Organization (WHO)/ International Society of Hypertension (ISH) statement on management of hypertension. J Hypertens 2003, 21(11):1983-1992.

32. Purcell $S$, Neale B, Todd-Brown K, Thomas L, Ferreira MAR, Bender D, Maller J, Sklar P, de Bakker PIW, Daly MJ, et al: PLINK: a tool set for wholegenome association and population-based linkage analyses. The Am J Human Genetics 2007, 81(3):559-575.

33. Barrett JC, Fry B, Maller J, Daly MJ: Haploview: analysis and visualization of LD and haplotype maps. Bioinformatics 2005, 21(2):263-265.

34. Burkett K, Graham J, McNeney B: Hapassoc: software for likelihood inference of trait associations with SNP haplotypes and other attributes. J Stat Softw 2006, 16(2):1-19.
35. Dahabreh IJ, Kitsios GD, Kent DM, Trikalinos TA: Paraoxonase 1 polymorphisms and ischemic stroke risk: a systematic review and meta-analysis. Genet Med 2010, 12(10):606-615.

36. Leviev N, Righetti A, James RW: Paraoxonase promoter polymorphism T $(-107) C$ and relative paraoxonase deficiency as determinants of risk of coronary artery disease. J Mol Med 2001, 79(8):457-463.

37. Deakin S, Leviev I, Brulhart-Meynet MC, James RW: Paraoxonase-1 promoter haplotypes and serum paraoxonase: a predominant role for polymorphic position-107, implicating the Sp1 transcription factor. Biochem Jl 2003, 372:643-649.

38. Demirdogen BC, Demirkaya S, Turkanoglu A, Bek S, Arinc E, Adali O: Analysis of paraoxonase 1 (PON1) genetic polymorphisms and activities as risk factors for ischemic stroke in Turkish population. Cell Biochem Funct 2009, 27(8):558-567.

39. Karakaya A, Ibis S, Kural T, Kose SK, Karakaya AE: Serum paraoxonase activity and phenotype distribution in Turkish subjects with coronary heart disease and its relationship to serum lipids and lipoproteins. Chemico-Biol Interact 1999, 118(3):193-200.

40. Brophy VH, Hastings MD, Clendenning JB, Richter RJ, Jarvik GP, Furlong CE: Polymorphisms in the human paraoxonase (PON1) promoter. Pharmacogenetics 2001, 11(1):77-84.

41. Brophy VH, Jampsa RL, Clendenning JB, McKinstry LA, Jarvik GP, Furlong CE: Effects of 5 ' regulatory-region polymorphisms on paraoxonase-gene (PON1) expression. Am J Hum Genetics 2001, 68(6):1428-1436.

42. Hofer SE, Bennetts B, Chan AK, Holloway B, Karschimkus C, Jenkins AJ, Silink M, Donaghue KC: Association between PON 1 polymorphisms, PON activity and diabetes complications. Jf Diabetes Complicats 2006, 20(5):322-328.

43. Leviev I, James RW: Promoter polymorphisms of human paraoxonase PON1 gene and serum paraoxonase activities and concentrations. Arterioscler Thromb Vasc Biol 2000, 20(2):516-521.

44. Freedman ML, Reich D, Penney KL, McDonald GJ, Mignault AA, Patterson N, Gabriel SB, Topol EJ, et al: Assessing the impact of population stratification on genetic association studies. Nat Genet 2004, 36(4):388-493.

45. Du R: Genetics of Chinese population. Beijing Sci Publish 2004, 10:414-419.

46. Wang X, Fan Z, Huang J, Su S, Yu Q, Zhao J, Hui R, Yao Z, Shen Y, Qiang B, et al: Extensive association analysis between oolymorphisms of PON gene cluster with coronary heart disease in Chinese Han population. Arterioscle Tthromb Vasc Biol 2003, 23(2):328-334.

47. Lash TL, Lien EA, Sørensen HT, Hamilton-Dutoit S: Genotype-guided tamoxifen therapy: time to pause for reflection? Lancet Oncol 2009, 10(8):825-833.

doi:10.1186/1471-2350-14-16

Cite this article as: Zhang et al:: Association between paraoxonase gene and stroke in the Han Chinese population. BMC Medical Genetics 2013 $14: 16$.

\section{Submit your next manuscript to BioMed Central and take full advantage of:}

- Convenient online submission

- Thorough peer review

- No space constraints or color figure charges

- Immediate publication on acceptance

- Inclusion in PubMed, CAS, Scopus and Google Scholar

- Research which is freely available for redistribution 